\title{
Montreal Cognitive Assessment Total Score
}

National Cancer Institute

\section{Source}

National Cancer Institute. Montreal Cognitive Assessment Total Score. NCI Thesaurus.

Code C162720.

The total score received in a Motreal Cong itive Assessment test. 